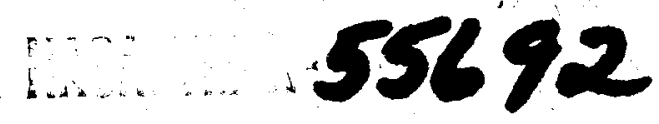

\title{
OGO-E COSMIC RADIATION - NUCLEAR ABUNDANCE EXPERIMENT
}

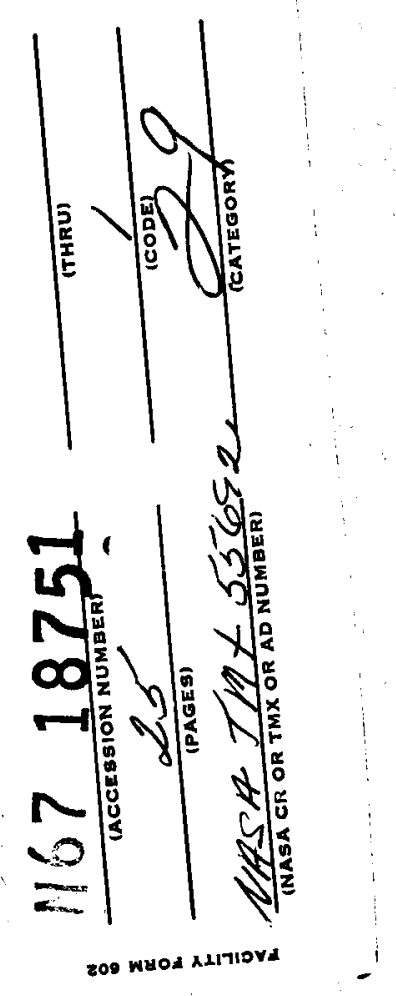

\author{
BY \\ S. L. JONES \\ G. H. LUDWIG \\ D. E. STILWELL \\ J. H. TRAINOR \\ S. H. WAY
}

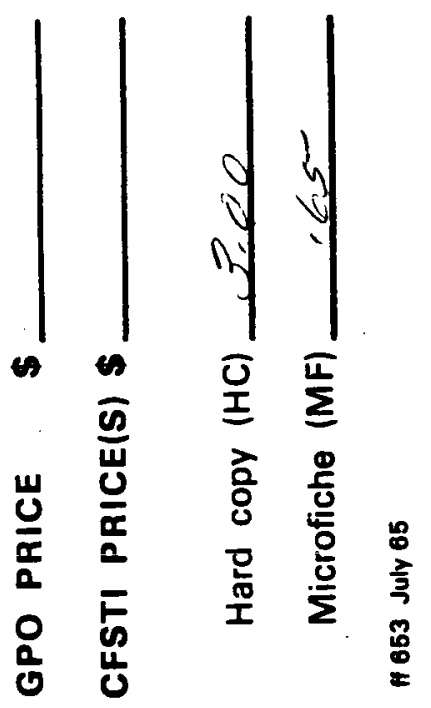

NASA

GODDARD SPACE FLIGHT CENTER

\section{GREENBELT, MARYLAND}

NOVEMBER 1966

Presented at the IEEE 13th Nuclear Science Symposium, Boston, Massachusetts; October 19 through 21, 1966. 
TABLE OF CONTENTS

Abstract . . . . . . . . . . . . . . . . . . . 1 Detector Systems

1. High Energy Detector . . . . . . . . . . . . 2

2. Medium Energy Detector . . . . . . . . . . . 2

3. Low Energy Detector . . . . . . . . . . . . . 2 Detector Electronics

1. High Energy Electronics System . . . . . . . . . 3

2. Medium Energy Electronics System . . . . . . . . 4

3. Low Energy Electronics System... . . . . . . . 5 Digital System . . . . . . . . . . . . . . . . 6 Reference ......................... 9 Figure Captions . . . . . . . . . . . . . . . . 9 
OGO-E Cosmic Radiation - Nuclear Abundance Experiment*

by

S. L. Jones, G. H. Ludwig, D. E. Stilwell, J. H. Trainor and S. H. Way

NASA/Goddard Space Flight Center

Greenbelt, Maryland

\begin{abstract}
$\underline{\text { Abstract }}$
Information on the detailed charge and energy spectra of galactic and solar cosmic rays is required for a better understanding of the production, acceleration and modulation of those cosmic rays. This experiment consists of a set of three detector assemblies to measure the differential energy spectra of protons and heavier nuclei through calcium $(Z=20)$ in the range of $400 \mathrm{Kev}$ to $1 \mathrm{Bev} /$ nucleon. This includes an extensive electronics system to implement the logic, perform pulse height analysis, measure particle fluxes, monitor experiment performance and interface with the OGO data system. The detector systems described here are the design of F. B. McDonald, V. K. Balasubrahmanyan, D. E. Hagge, B. Teegarden and G. H. Ludwig of the Goddard Space Flight Center.
\end{abstract}

* Presented at the IEEE 13th Nuclear Science Symposium, Boston, Massachusetts, October 19 through 21, 1966. 
DETECTOR SYSTEMS

\section{High Energy Detector:}

Figure 1 shows the highest energy detector assembly schematically and lists the major logic involved. Scintillators $A$ and $B$ are CsI(T1) while the Cerenkov radiator is the one $\mathrm{cm}$ thick sapphire window of the photomitiplier tube. $A \overline{B C}$ events above appropriate thresholds define the passage of a particle with energy from $\sim 50 \mathrm{Mev} /$ nucleon to $220 \mathrm{Mev} /$ nucleon. Similarly, $A B C$ logical events define particles with energies from $220 \mathrm{Mev} / \mathrm{nucleon}$ to $\sim 1000 \mathrm{Mev} / \mathrm{nucleon}$ and the integral flux of particles with energies greater than $\sim 1000 \mathrm{Mev} /$ nucleon. Measurements of the two scintillation and the Cerenkov detector pulse heights identifies the charge and energy of the incident particle over the indicated ranges.

\section{Medium Energy Detector:}

This detector assembly and its logic are shown in Figure 2. D and $F_{\text {slow }}$ are thin dE/dx CsI(T1) scintillators while E is a thick "tota1 energy" CsI scintillator. Ffast is a plastic guard scintillator which encloses $E$. A $D E \bar{F}_{\text {fast }}$ event enables pulse height analysis of the $D$, $\mathrm{E}$ and $\mathrm{F}_{\mathrm{S} \text { low }}$ pulses which will identify charge and energy of the particle from $20 \mathrm{Mev} / \mathrm{nucleon}$ to $\sim 300 \mathrm{Mev} /$ nucleon. All events in the D scintillator are analyzed in a fast, three step, integral pulse height analyzer to provide flux and energy information during high flux solar proton events.

\section{Low Energy Detector:}

This telescope is shown in Figure 3 and is composed of large area, totally depleted, surface barrier solid state detectors surrounded by a 
guard scintillator. Detector $G$ is 100 microns thick while $H$ consists of two 1000 micron detectors with the outputs summed. GHJ logic defines events due to particles with energies from $3.5 \mathrm{Mev} / \mathrm{nucleon}$ to $18.5 \mathrm{Mev} /$ nucleon, while five integral discrimination levels on G provide flux. and energy information for solar flare events.

Figure 4 is a picture of these detector assemblies, including the high energy detector in one half of its flight cradle. Each assembly includes the detectors, photomultiplier tubes, preamplifiers, the Cockroft-Walton multipliers on the base of each PM tube and the high frequency oscillator which drives the multipliers.

\section{DETECTOR ELECTRONICS}

\section{High Energy Electronics System:}

Figure 5 depicts the two 256 channel and one 400 channel differential pulse height analyzers with associated coincidence and gain changing circuitry which make up this electronics system. Most of these circuits were discussed in detail in a previous paper by Way, Stilwell, and Jones (1966). Each pulse height analyzer is basically identical with slight differences in sensitivities to give the desired dynamic ranges. The sensitivities of the $A$ and $B$ analyzers are 1.0 and 12.0 millivolts per channel in the high and low gain modes respectively, while those of the $C$ analyzer are 0.5 and 4.0 millivolts per channel.

The decision to change gain from one mode to another (high to low gain) is done on an individual event basis with the $A$ and $B$ analyzers changing together and $\mathrm{C}$ changing independently. A pulse summing circuit 
fed by the $A$ and $B$ pulses determines the gain change point for $A$ and $B$, while the amplitude of the $\mathrm{C}$ pulse alone determines if it is to be analyzed in high or low gain.

The coincidence circuit is a simple combination of monostables, tunnel diodes AND and INHIBIT logic circuits (Way, Stilwe11 and Jones, 1966). The resolution is between 0.5 and 0.75 microseconds for rates up to 100,000 pps.

Measured performance of the electronics is given briefly below: Integra1 linearity, analyzer - less than $1 \%$ over top $95 \%$ of scale; temperature stability, analyzers - less than $\pm 0.5 \%,+5^{\circ} \mathrm{C}$ to $+40^{\circ} \mathrm{C}$; zero level offset - one channe1; thresholds, A and B, adjustable channe1 2.0 to channel 2.5; power consumption, al1 electronics - 250 milliwatts. Figure 6 is a picture of an electronics assembly containing three pulse height analyzers.

\section{Medium Energy Electronics System:}

Three 256 channel differential pulse height analyzers, one three level integral pulse height analyzer, coincidence, gain changing and pulse shape discriminating, anticoincidence circuitry make up these electronics as shown in Figure 7. Each differential pulse height analyzer is identical. The basic high gain sensitivity of each analyzer is one millivolt per channel and the low gain sensitivity is sixteen millivolts per channel. The maximum dynamic range in either high or low gain is 256 which is determined by the accumulating capacity of the digital data processor. 
The integral analyzer is a simple tunnel diode discriminator circuit fed by a stair step current generator (Figure 8). Drive for each of the ladder-adder resistors is from a string of commutator binaries to be described with the digital data system. The first level threshold and the following level widths are adjusted by resistor value. Triggering levels on the order of 10 millivolts are typical for the bottom level.

The coincidence circuit is basically the same as for the high energy electronics with the addition of the anticoincidence logic. The pulse shape discriminating circuit is necessary to separate the pulses from the plastic guard scintillator and the Cesium Iodide (T1) scintillator. This is done by detecting the pulse peak and generating an anticoincidence pulse at that time. The timing in the coincidence circuitry is such that only a plastic scintillation will produce an anticoincidence pulse soon enough to prevent an analysis of the event.

\section{Low Energy Electronics System:}

The system shown in Figure 9 provides for a dE/dx vs. E particle analysis using two 256 channel pulse height analyzers and a 200 nanosecond coincidence resolving time. Energy losses between $200 \mathrm{Kev}$ and $13 \mathrm{Mev}$ in the $\mathrm{G}$ detector, between $800 \mathrm{Kev}$ and $77 \mathrm{Mev}$ in the $\mathrm{H}$ detector and not greater than about $200 \mathrm{Kev}$ in the guard scintillator are required for pulse height analysis. A five level integral analyzer similar to that previously described is included to monitor the large fluxes present during solar flare events. 
Fal1 times of the $G$ and $H$ charge sensitive preamplifiers are set for $\sim 10$ microseconds to insure that charge collection time is maximum. The active RC shapers provide pulse pile up rejection for these two channels (Way, Stilwe11 and Jones, 1966). The fall time of the J charge sensitive preamplifier pulse can be clipped to 0.5 microsecond since a fast plastic scintillator is used, and thus additional shaping is not required to permit high counting rates.

The system linearity is $\pm 2.5 \%$ over the specified energy ranges, and over the temperature range of $-10^{\circ} \mathrm{C}$ to $+50^{\circ} \mathrm{C}$. Signal limiters are placed in the amplifying chains to minimize amplifier dead time due to high level signals. The coincidence circuitry utilizes non-saturating, tunnel diode input, bootstrap amplifiers and fast recovery time monostables. The entire system is constructed in welded-wire modules using a printed circuit board for the interconnect.

Calibration of the detectors and electronics is performed with a Van de Graaff accelerator using the $\mathrm{He}^{3}$ on $\mathrm{D}^{2}$ reaction to obtain protons up to $17 \mathrm{Mev}$. Proton energy is varied at the detector window by inserting aluminum foil absorbers of known thickness in the beam path .

\section{DIGITAL SYSTEM}

The digital system accepts PHA pulse trains and rate information from the detectors as shown in the block diagram (Figure 10) and presents the information to the OGO central telemetry system as serial NRZ data in accordance with the word and bit signals received from the 
spacecraft. The digital system, shown in greater detail in Figure 11, consists basically of two types of counters (shown as 9-bit counters and a floating-point accumulator, or FPA), control and readout modules, a multiplexer and a commutator. The control, readout and multiplexer modules are redundant in that each of these boxes on the block diagram corresponds to two physically separate but identical modules of the appropriate type. To conserve power, operating voltages are app1ied only to one set of modules in a given operating mode. This is represented by the "inputs" designated Power I and Power II.

Each of the detectors $\mathrm{A}$ through $\mathrm{H}$ are associated with a given pulse height analyzer and 9-bit counter with corresponding designations. The counter may be operated either as an 8-bit counter plus a single "set" register or as a conventional 9-bit counter by suitable connections on the plug-in card. Only detector $\mathrm{C}$ uses the 9-bit configuration, a11 remaining detectors use 8 bits for 256 channels of pulse height analysis, and the 9 th bit to indicate one of two possible conversion gains or to provide information about the multiplexer and commutator positions. The quasi-floating point accumulator consists of 5 counting stages and 4 prescaler-selecting binaries to select one of 16 stages of prescaling. The total count capacity of this configuration is $2,097,119$ and the maximum error is 1 part in 32 , or just over $3 \%$.

The 16 position commutator sequentially selects one of eight detector rates or one of eight integral analyzer rates to be counted by the floating point accumulator. The integral analyzer level is selected by the commutator binaries as previously described. 
The system configuration accepts eight inhibit signals from one of the two spacecraft data encoders. The eight words are divided into four groups of two adjacent words each and directed to the proper counters to provide the read-out format shown in Figure 12. Each counter readout is repeated to permit detection of data containing probable bit errors, and to provide redundancy in the event of any control circuit failures.

The data processor was developed under contract with General Instruments, Inc. (Hicksville, N. Y.) using multiple chip microcircuit technology. Discreet silicon chips for all active and passive components are alloyed to a ceramic substrate and interconnected by thermo-compression bonded 1 mil diameter solid gold wire. Each such flatpack contains a complete circuit function (e.g., amplifiers or flip flop) consisting of up to 40 chips. The flat packs are packaged in cordwood style and solidpotted in epoxy to form the functional modules shown in the block diagram. The complete data processing system, shown in Figure 13, contains over 2000 active devides on a single $51 / 2 \times 71 / 2 \times 1$ inch plug-in card. The circuitry was designed to operate properly with from 4.5 to 9.0 volts supply voltage and throughout the temperature range $-40^{\circ} \mathrm{C}$ to $+85^{\circ} \mathrm{C}$ at clocking speeds in excess of $1 \mathrm{M} \mathrm{Hz}$. Power consumption averages 2.3 mw/bit . 
Way, Stanley, D. Stilwel1 and S. L. Jones, "A Three Parameter Pulse Height Analyzer and Coincidence System for Satellite Cosmic Ray Studies," IEEE Trans. Nuc. Sci., NS-13, No. 1, 1966.

\section{FIGURE CAPTIONS}

Figure 1: $\quad$ Schematic of the high energy detector

Figure 2: $\quad$ Schematic of the medium energy detector

Figure 3: Schematic of the low energy detector

Figure 4: Flight detector assemblies and cradles

Figure 5: Electronics for the high energy system

Figure 6: Partially assembled electronics board with three differential pulse height analyzers

Figure 7: $\quad$ Electronics for medium energy system

Figure 8: $\quad$ Basic integral pulse height analyzer

Figure 9: Electronics for low energy system

Figure 10: Block diagram of the entire experiment

Figure 11: The basic digital data processor

Figure 12: $\quad$ Experiment data format

Figure 13: The digital data processor packaged for space flight 

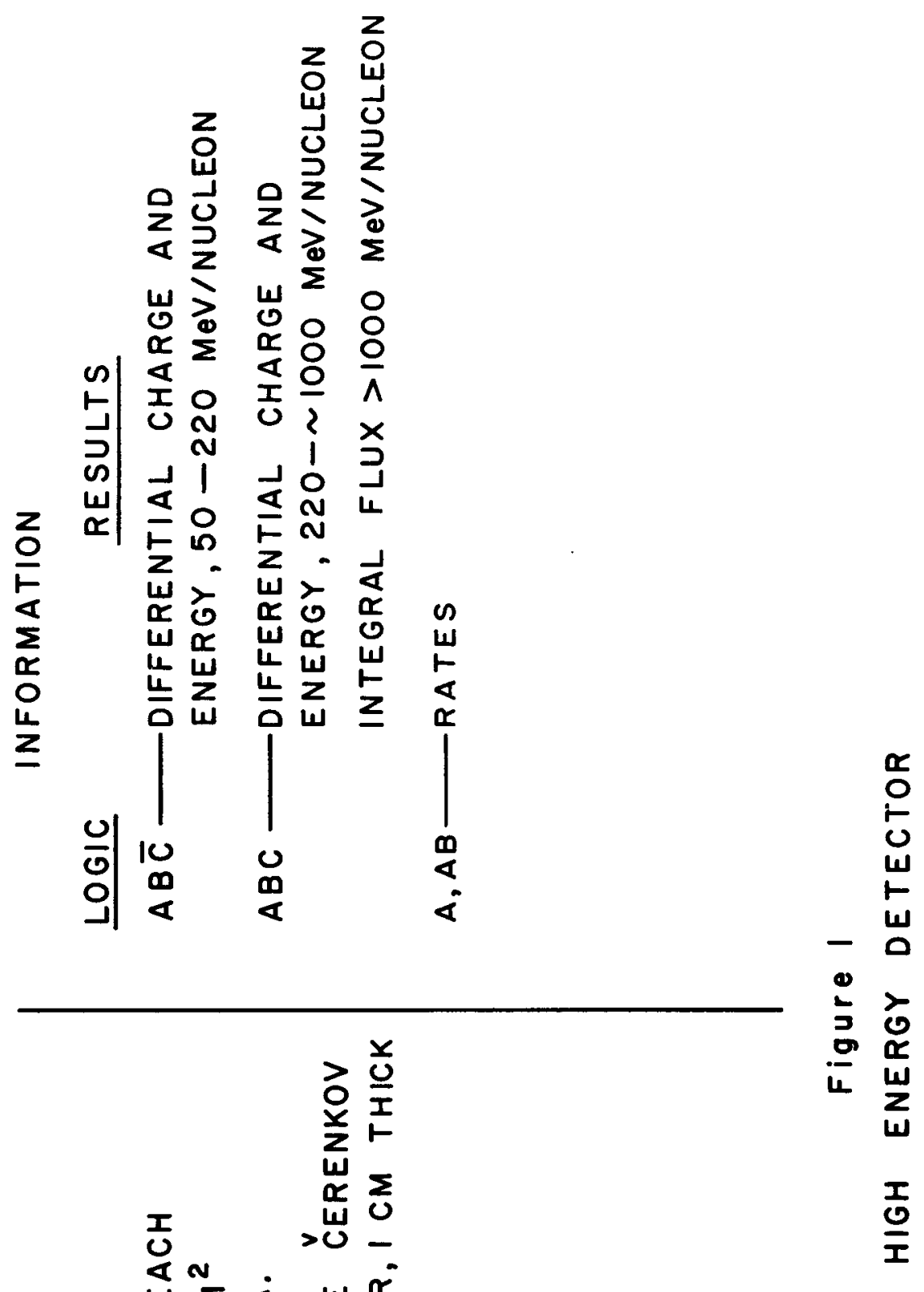


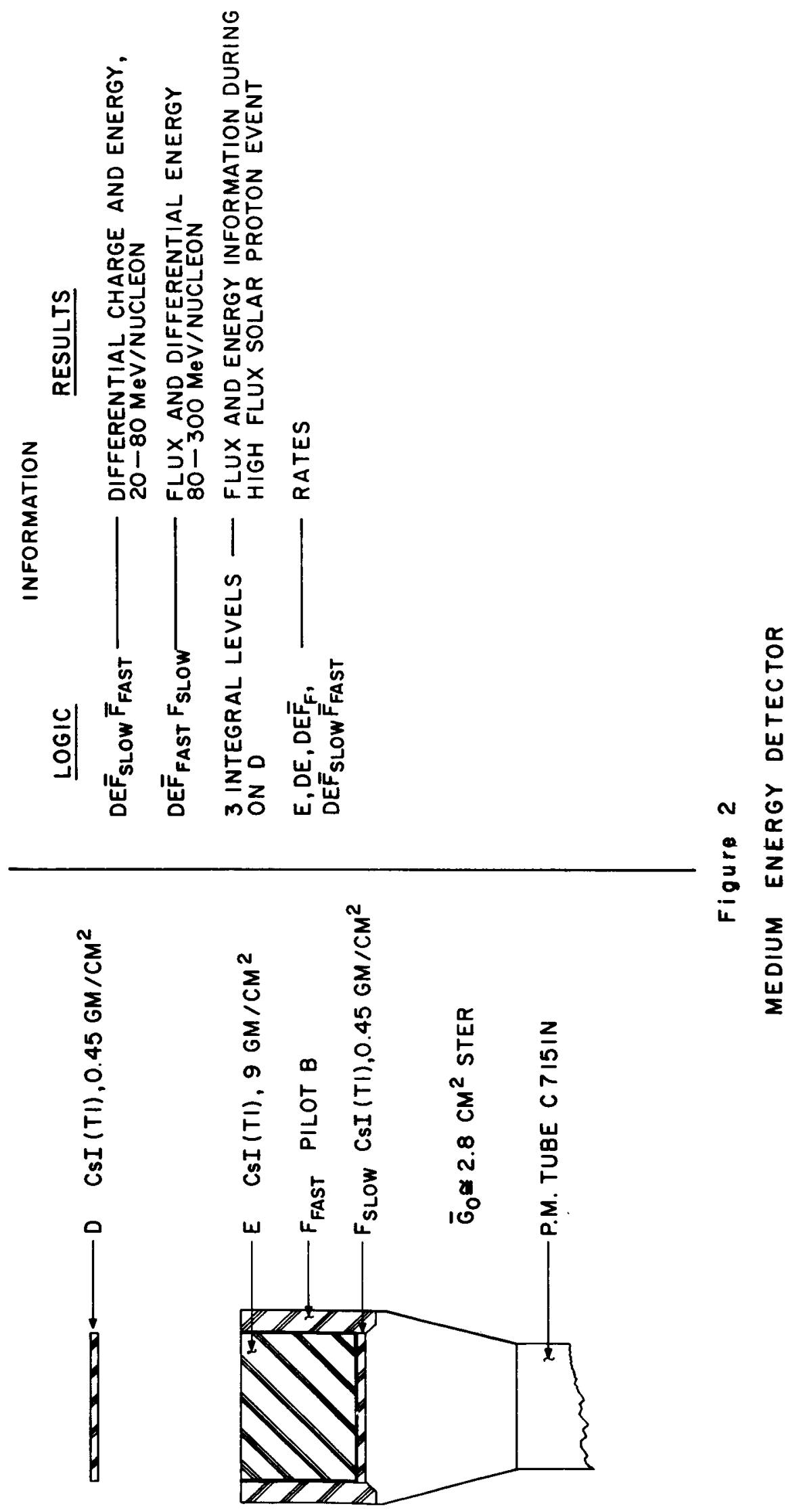




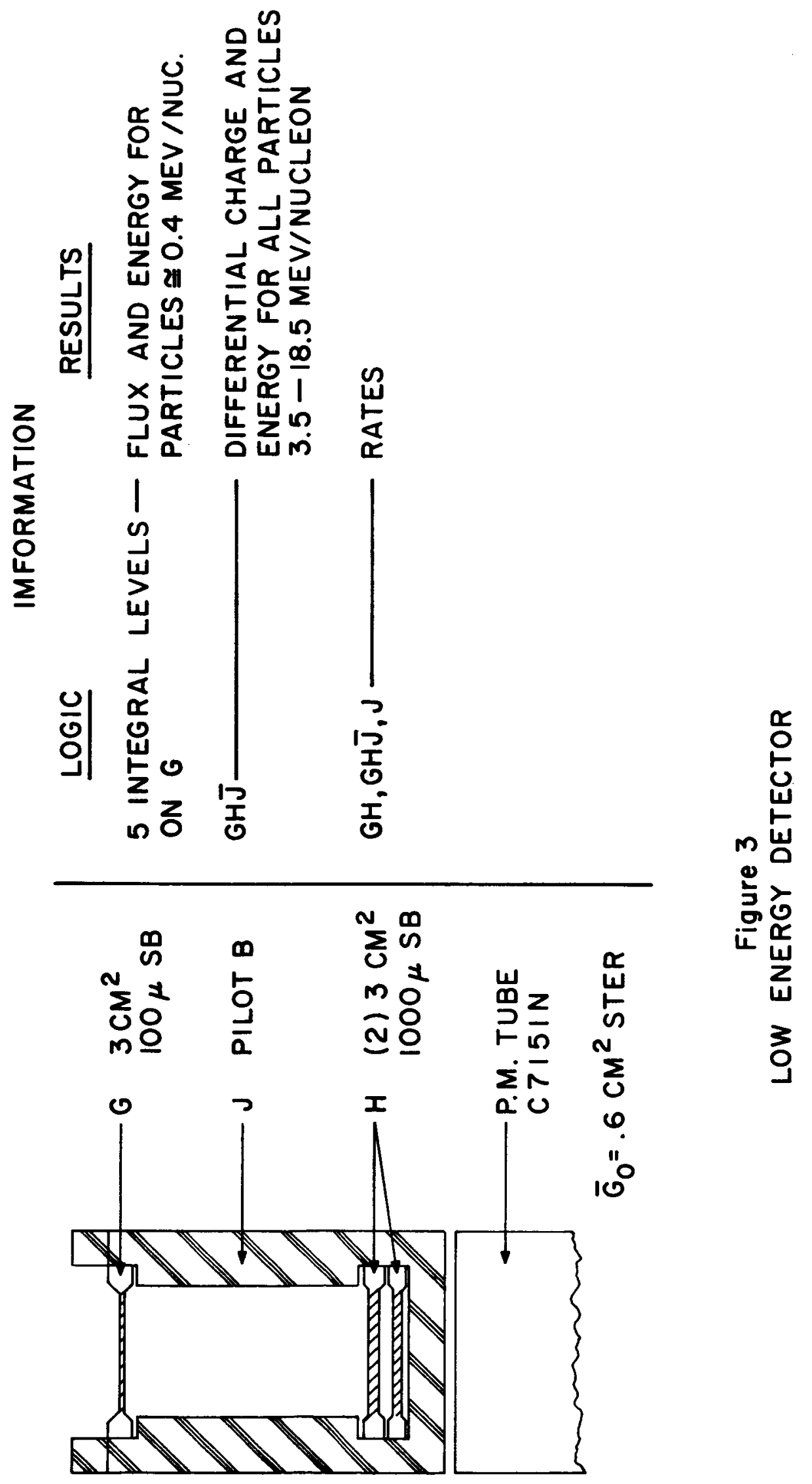




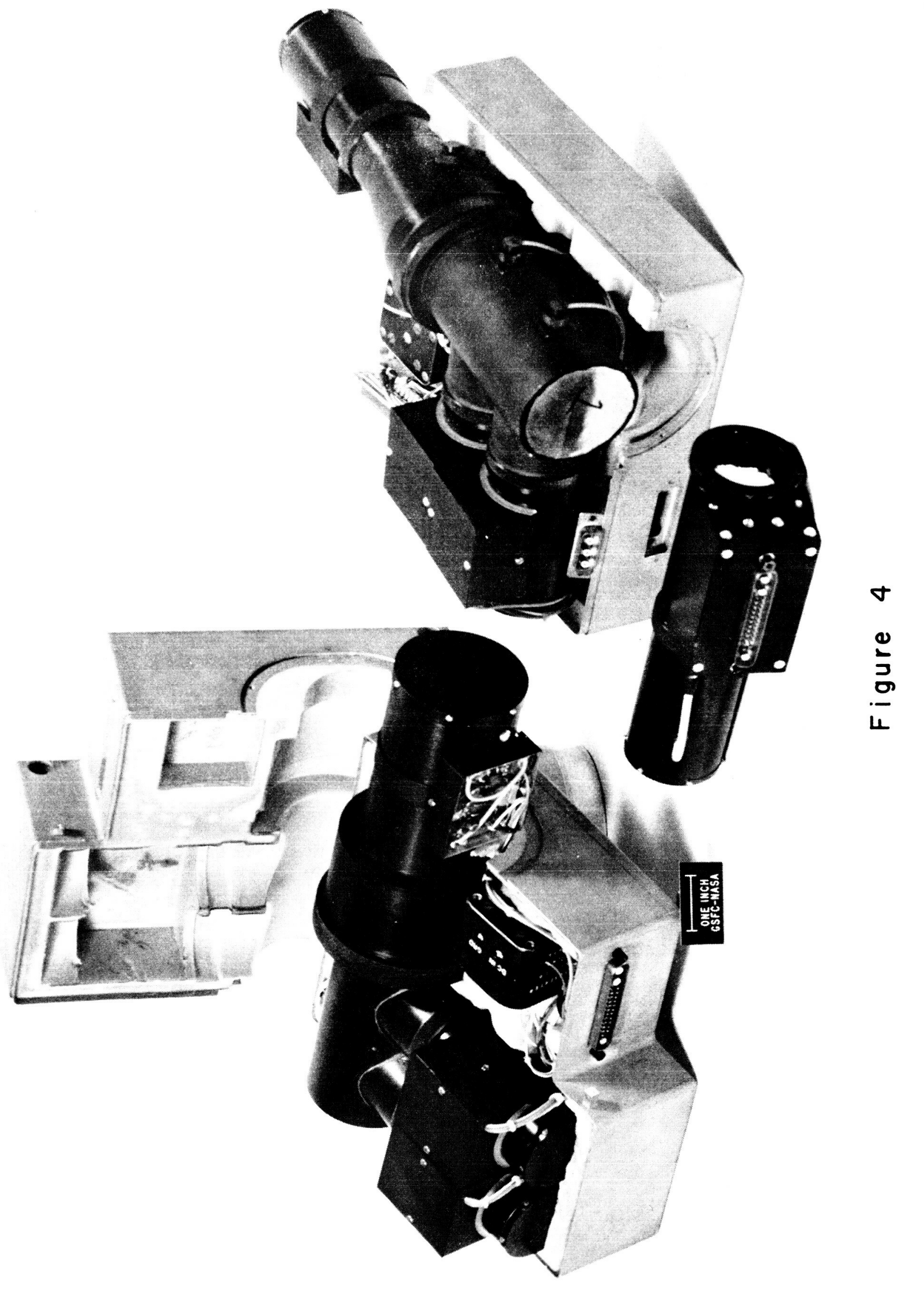




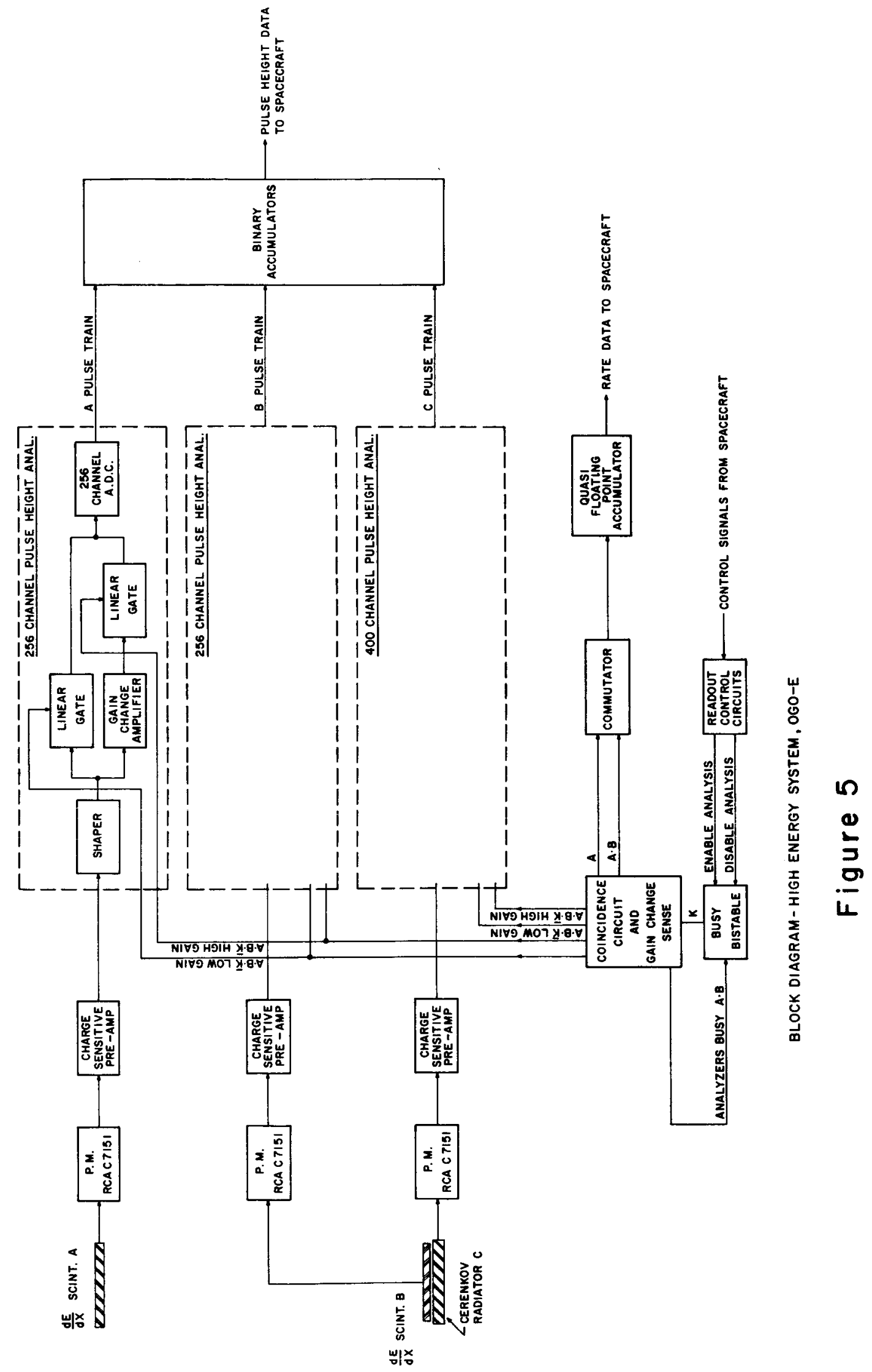




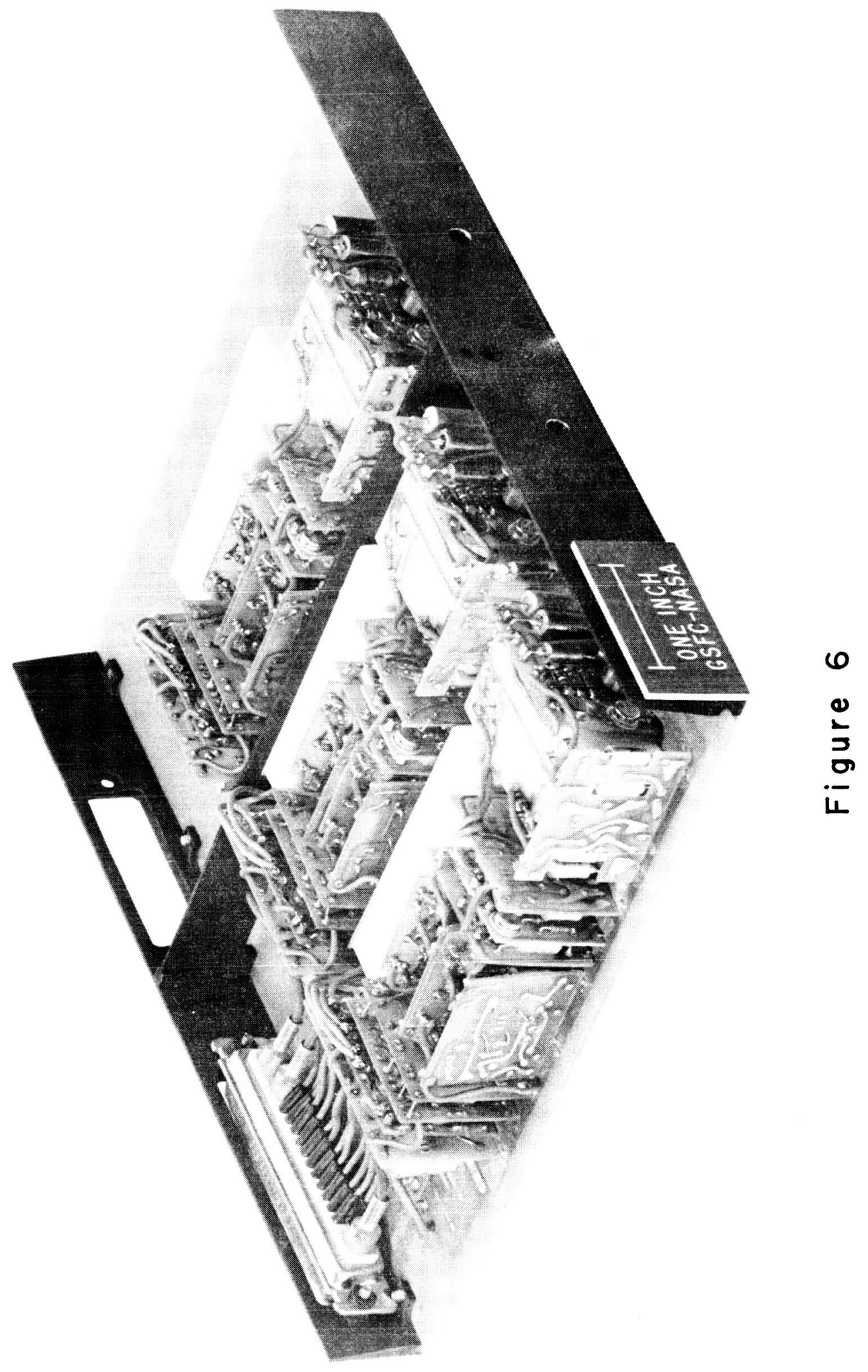




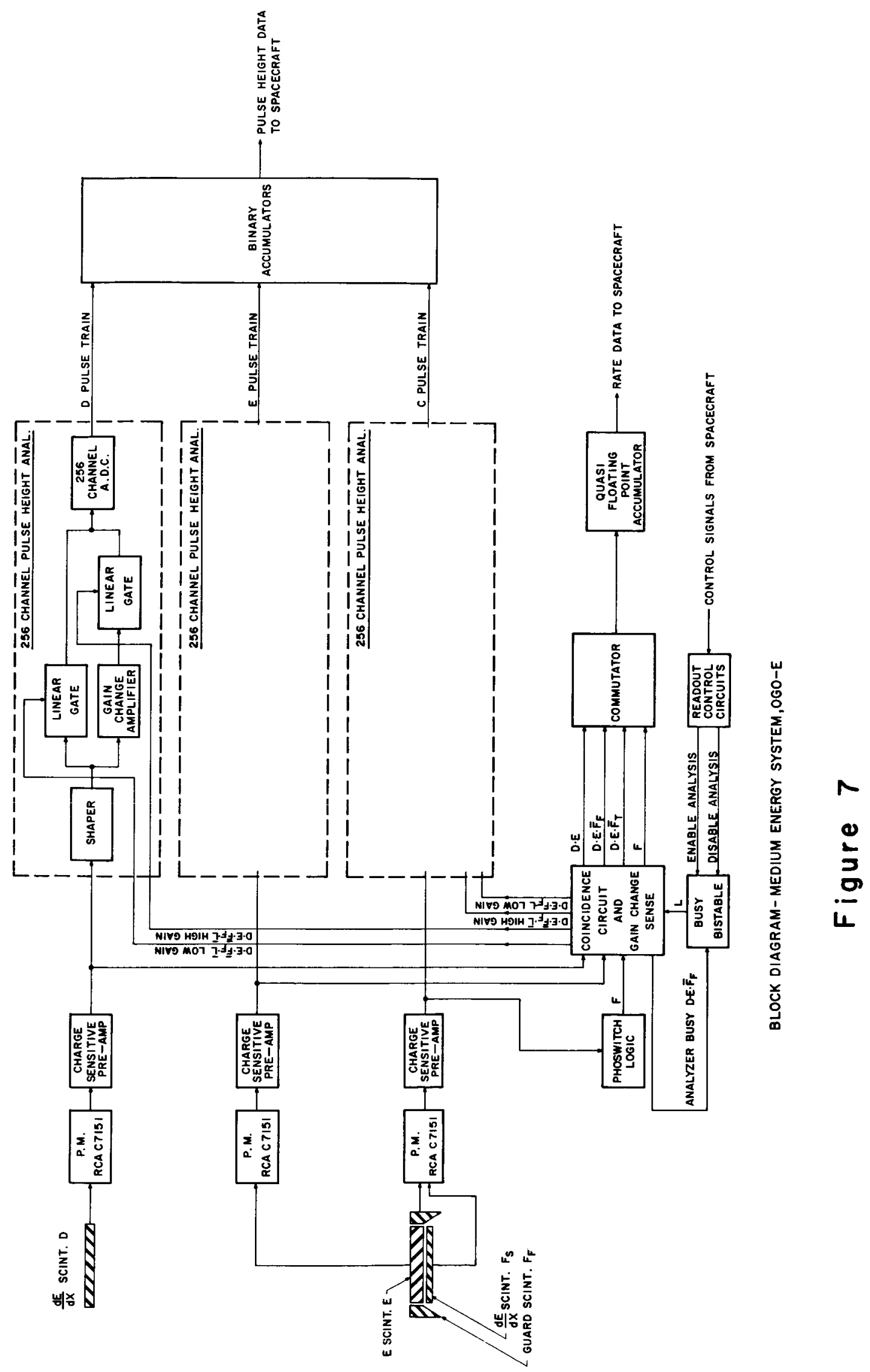




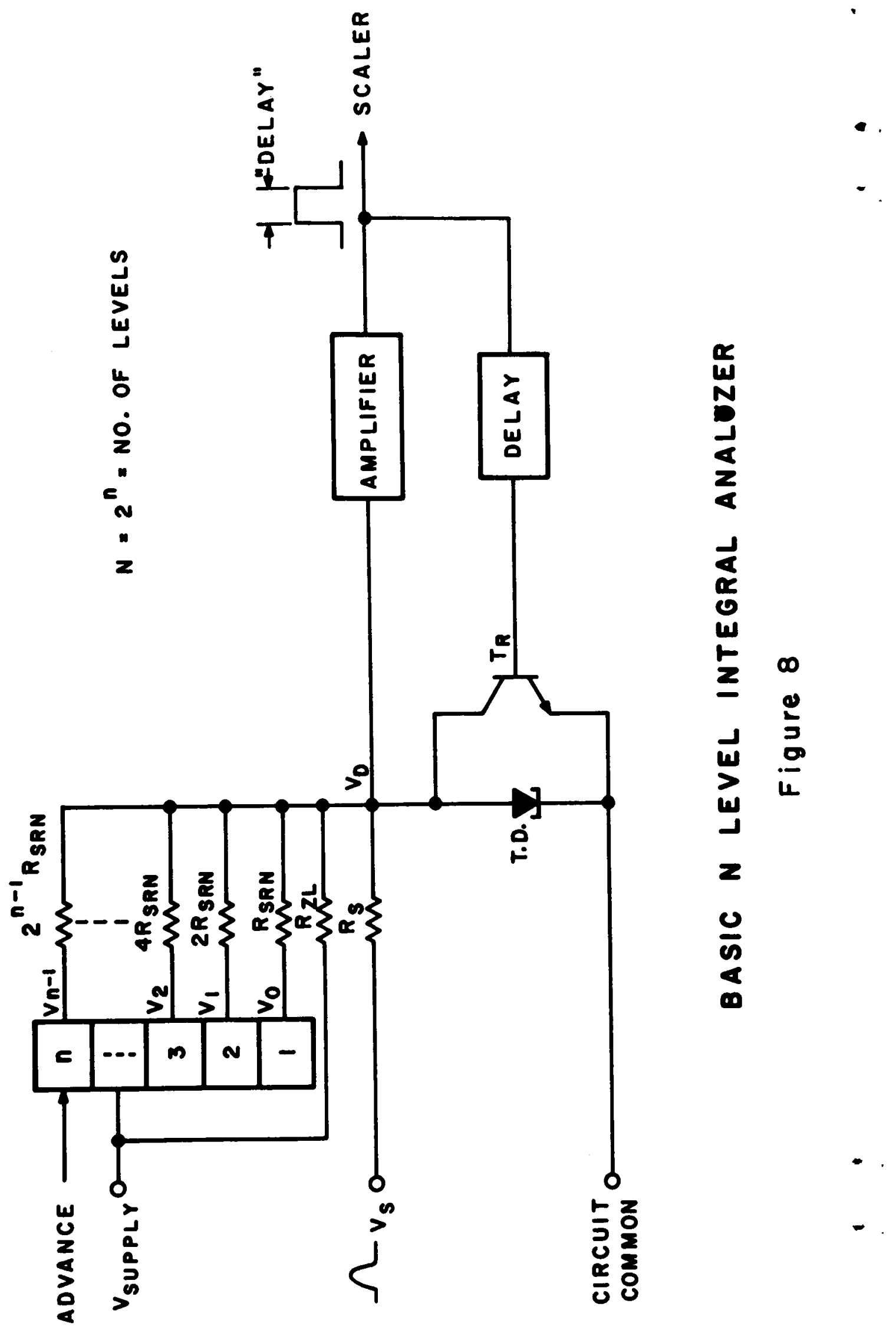




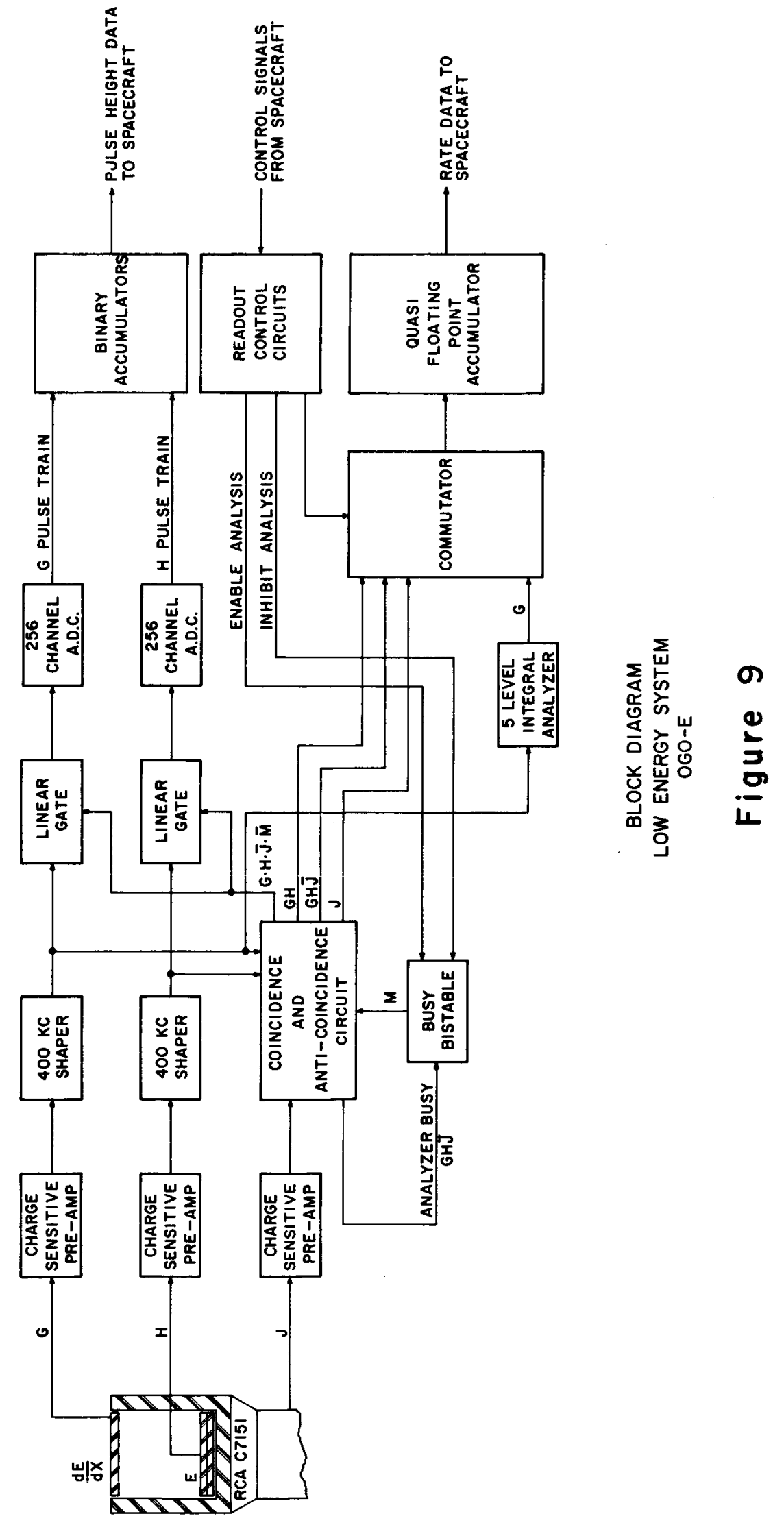




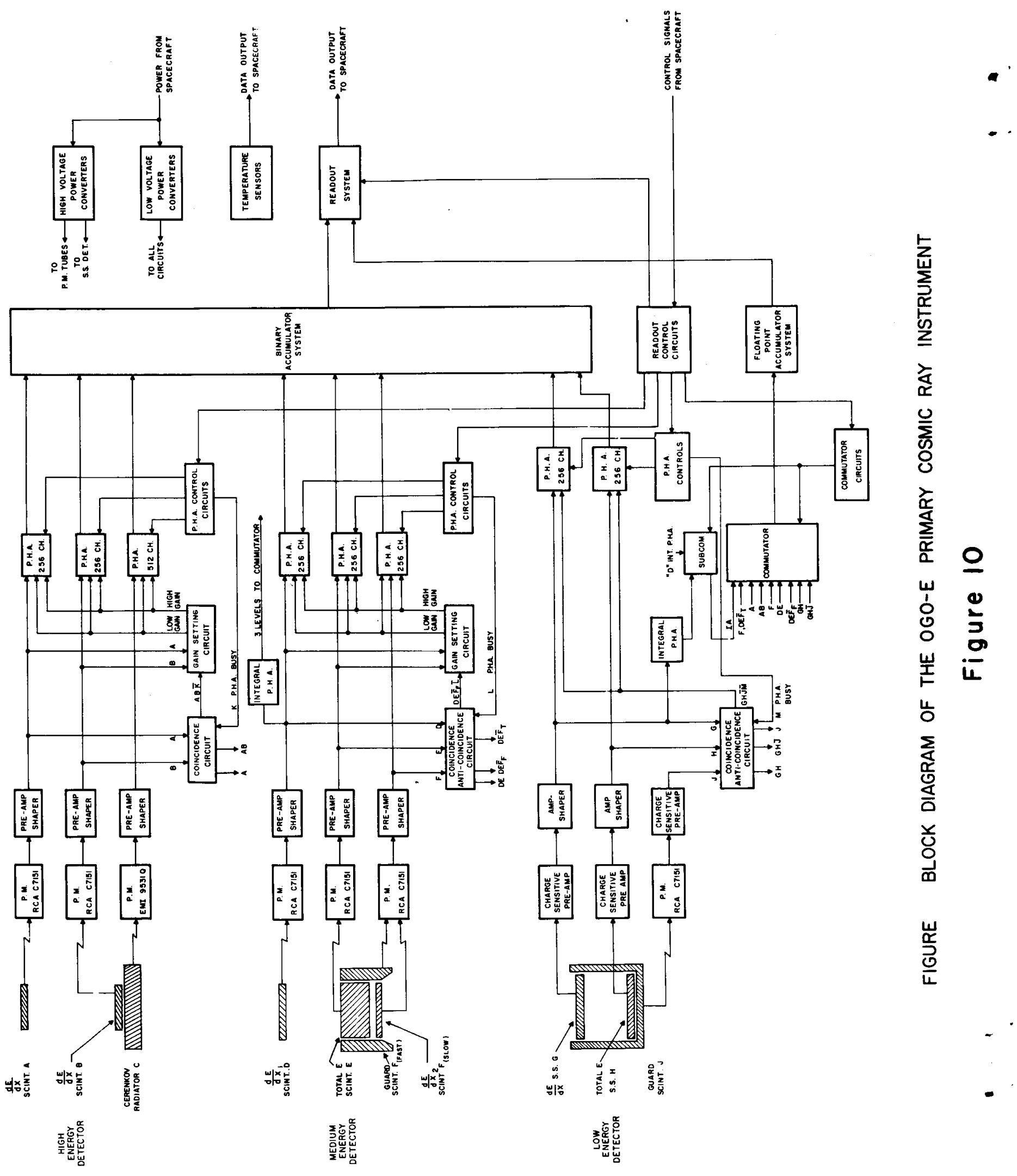




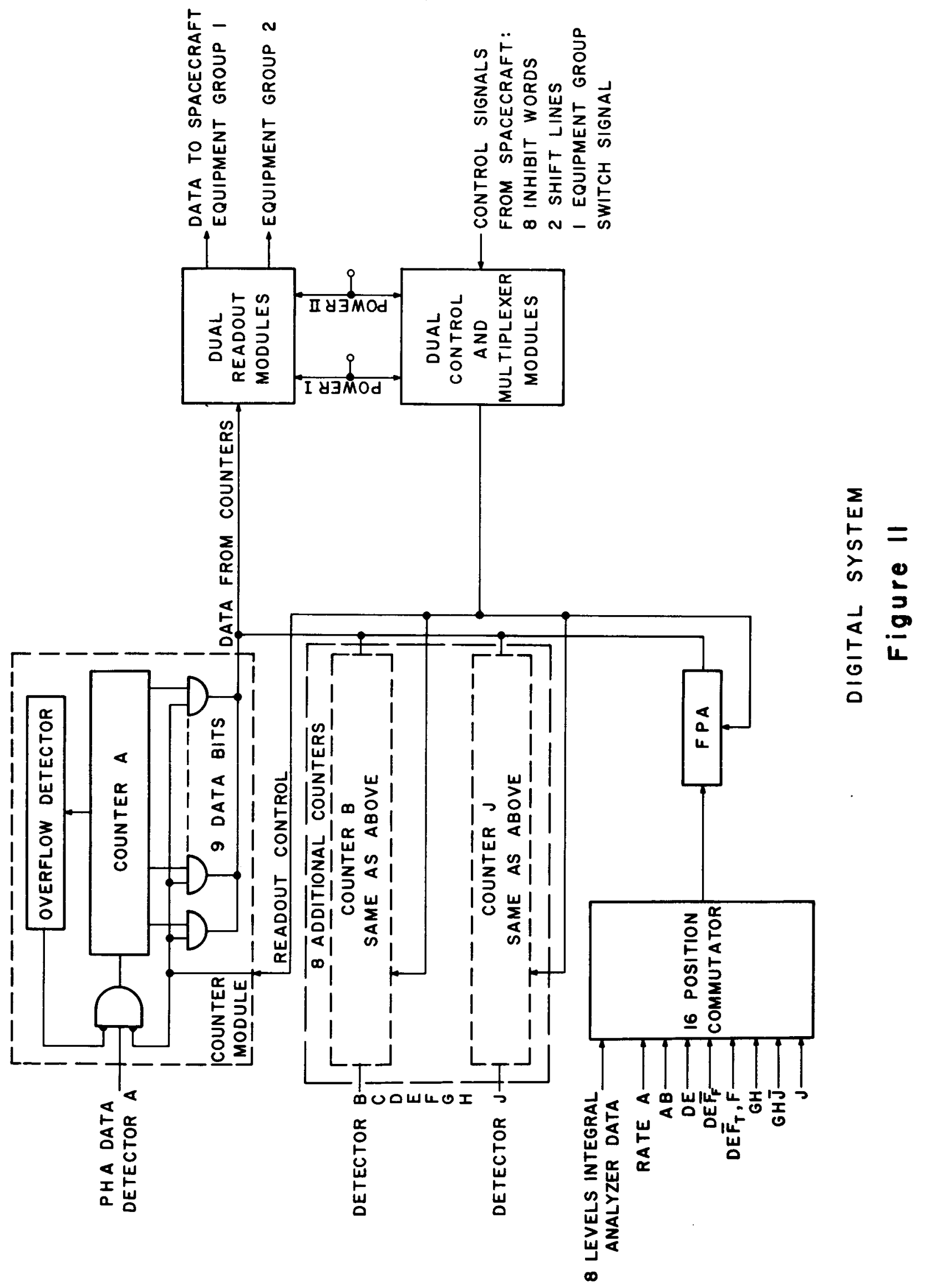




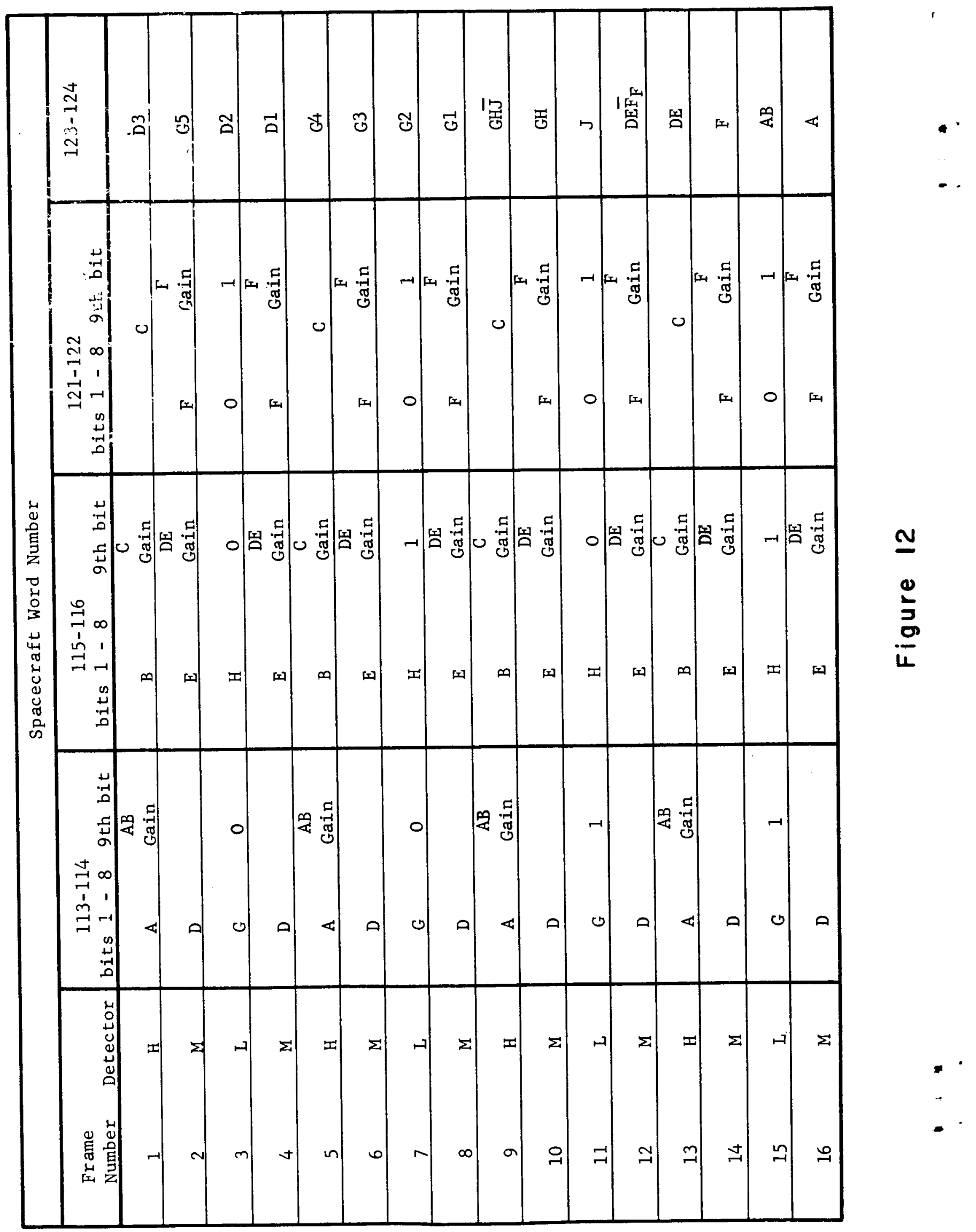




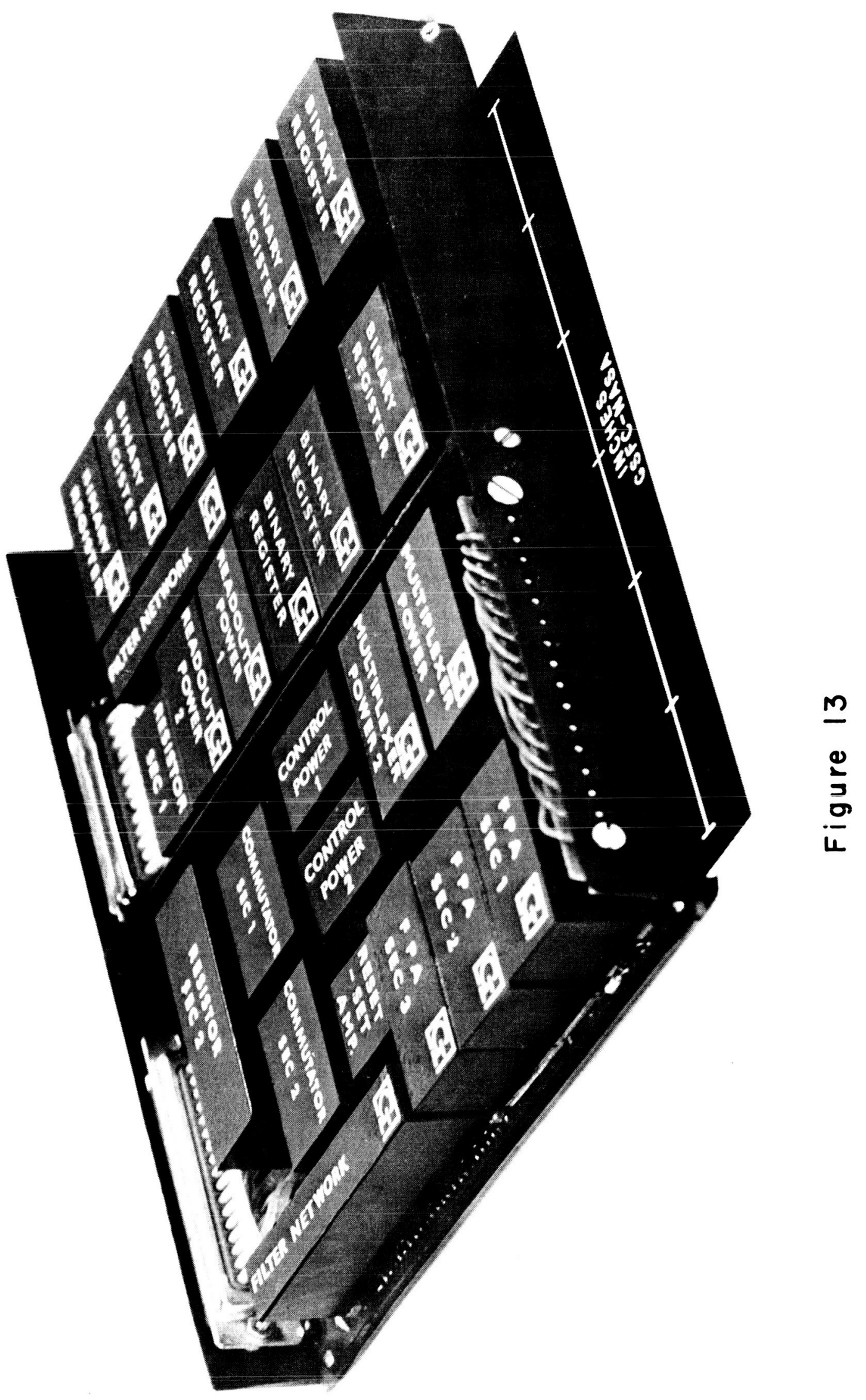

